\title{
SOME ASPECTS OF THE COMBINATORIAL THEORY OF CONVEX POLYTOPES
}

\author{
GIL KALAI \\ Institute of Mathematics \\ Hebrew University \\ Jerusalem, 91904, Israel \\ e-mail: kalai@humus.huji.ac.il
}

\begin{abstract}
.
We start with a theorem of Perles on the $k$-skeleton, $S k e l_{k}(P)$ (faces of dimension $\leq k$ ) of $d$ polytopes $P$ with $d+b$ vertices for large $d$. The theorem says that for fixed $b$ and $d$, if $d$ is sufficiently large, then $\operatorname{Skel}_{k}(P)$ is the $k$-skeleton of a pyramid over a $(d-1)$-dimensional polytope. Therefore the number of combinatorially distinct $k$-skeleta of $d$-polytopes with $d+b$ vertices is bounded by a function of $k$ and $b$ alone. Next we replace $b$ (the number of vertices minus the dimension) by related but deeper invariants of $P$, the $g$-numbers. For a $d$-polytope $P$ there are $[d / 2]$ invariants $g_{1}(P), g_{2}(P), \ldots, g_{[d / 2]}(P)$ which are of great importance in the combinatorial theory of polytopes. We study polytopes for which $g_{k}$ is small and carried away to related and slightly related problems.

Key words: Convex polytopes, skeleton, simplicial sphere, simplicial manifold, $f$-vector, $g$ theorem, ranked atomic lattices, stress, rigidity, sunflower, lower bound theorem, elementary polytopes
\end{abstract}

\section{Introduction}

\subsection{Overview}

In this paper we will discuss several combinatorial problems concerning the combinatorial structure of polytopes. For a $d$-polytope $P$ the number of $k$-faces is denoted by $f_{k}(P)$. The vector $\left(f_{0}(P), f_{1}(P), \ldots f_{d-1}(P)\right)$ is called the $f$-vector of $P$. The same definitions will apply to more general combinatorial objects considered below. The $k$-th skeleton $\operatorname{Skel}_{k}(P)$ of a $d$-polytope $P$ is the set of all faces of $P$ of dimension $\leq k$. $\operatorname{Skel}_{1}(P)$ is called the graph of $P$ and is denoted by $G(P)$.

A simple basic fact is that for every $d$-dimensional polytope $P$

$$
f_{0}(P) \geq d+1
$$

Equality holds if and only if $P$ is a simplex. Important part of convex polytope theory is the study of polytopes with "few vertices", namely polytopes with a bounded difference between the nunber of vertices and the dimension. The following theorem of Perles is part of the theory of polytopes with "few vertices" and it will play a central role in this paper.

Theorem 1.1 (Perles, 1970) Let $f(d, k, b)$ be the number of combinatorial types of $k$-skeleta of $d$-polytopes with $d+b+1$ vertices. Then for fixed $b$ and $k, f(d, k, b)$ is bounded. 
A proof of Perles theorem is given in Section 2. (The proof relies only section 1.4 from the Introduction.) The proof given here is somewhat different from Perles' original proof. It relies, like the original proof on the important concept of missing faces. The proof here uses the famous sunflower (Delta-system) theorem of Erdös and Rado.

A construction which increase by one both the dimension and the number of vertices is forming a pyramid over a polytope. Perles theorem asserts that for fixed $b$ and $d$, if $d$ is sufficiently large, then $\operatorname{Skel}_{k}(P)$ is the $k$-skeleton of a pyramid over a $(d-1)$-dimensional polytope. In contrast, note that the number of combinatorial types of $d$-polytopes with $d+3$ vertices is bounded below by an exponential function of $d$, see [24].

Another theorem which is basic to the discussion in the second part of this paper is the lower bound theorem which was conjectured by Brückner in 1909 and was proved by Barnette [7] in 1970.

Theorem 1.2 For every simplicial d-polytope $P$,

$$
f_{1}(P) \geq d f_{0}(P)-\left(\begin{array}{c}
d+1 \\
2
\end{array}\right) .
$$

Equality is obtained by stacked polytopes, namely polytopes built by gluing simplices along facets.

For a $d$-polytope $P$ there are $[d / 2]$ invariants $g_{1}(P), g_{2}(P), \ldots, g_{[d / 2]}(P)$ which are of great importance in the combinatorial theory of polytopes. $g_{1}(P)$ is just the difference between the number of vertices of $P$ and $d+1$. For simplicial polytopes $g_{2}(P)$ is the difference between the left hand side and the right hand side of the lower bound relation (2).

In analogy with the theory of polytopes with "few vertices" we discuss in Sections 3 and 4 the combinatorial properties of polytopes with a bounded value of $g_{k}$ for some fixed $k$. The nonnegativity of $g_{k}(P)$ is in general a deep fact (and for $k>2$ it is not even known for general polytopes which cannot be realized by vertices with rational coordinates.) And we try to use the methods originally applied to prove the nonnegativity of $g_{k}(P)$ to study those polytopes for which $g_{k}(P)$ is small.

Section 3 deals with simplicial polytopes and Section 4 deals with general polytopes. In both cases the case $k=2$ is substantially simpler than the general case. We will use in this discussion the notion of stresses and the connection between stresses and the $g_{k}$ 's as developed in Carl Lee's paper [2]. Our discussion in Section 4 is strongly related to the first section in Margaret Bayer's paper [1] and also to some topics in Richard Stanley's paper [3].

The paper is written in somewhat ununiform style. The discussion in Section 2 is self-contained and elementary. In Sections 3-4 while technically the paper is still mostly self-contained, some prior familiarity with the notions of $h$-vectors, the lower bound theorem, the $g$-theorem and the algebraic tools which play a role in their study would be very useful. There are many problems and conjectures which are quoted and stated all around the paper.

\subsection{A START}

Theorem 1.3 Every d-dimensional polytope has at least $d+1$ vertices. 
Proof 1 (geometric-algebraic) : This follows at once from the fact that the vertices of a $d$-dimensional polytope affinely span a $d$-dimensional space.

Proof 2 (combinatorial) By induction: Let $P$ be a $d$-polytope and let $F$ be a facet of $P$. By the induction hypothesis $F$ has at least $d$ vertices. There must be a vertex in $P$ not in $F$ therefore $P$ has at least $d+1$ vertices.

The combinatorial proof has the advantage that it applies to much more general combinatorial objects (ranked relatively-complemented lattices). The geometric proof show that $g_{1}(P)$ is the dimension of the space of affine relations among the vertices of $P$, and suggests to study polytopes with small value of $g_{1}$ by looking on the space of affine relations among vertices. This is the starting point of a very useful theory of "Gale diagrams" see [24] Ch. 6.

Both proofs show that equality holds if and only if $P$ is a simplex.

The combinatorial proof easily extends to prove the inequality

$$
g_{1}[r](P)=: f_{r}(P)-\left(\begin{array}{l}
d+1 \\
r+1
\end{array}\right) \geq 0 .
$$

Indeed, given a $d$-polytope $P$ and a facet $F$ of $P$, every $(r-1)$-face $G$ of $F$ is included in an $r$-face $H_{G}$ of $P$ such that $H_{G}$ itself in not contained in $F$. It follows that $H_{G} \cap F=G$, and therefore $G \rightarrow H_{G}$ is a one to one map from $(r-1)$-faces of $F$ to $r$-faces of $P$ which are not contained in $F$. Thus, by an induction hypothesis there are at least $\left(\begin{array}{c}d-1 \\ r\end{array}\right) r$-faces of $P$ contained in $F$, and at least $\left(\begin{array}{c}d-1 \\ r-1\end{array}\right) r$-faces of $P$ which are not contained in $F$.

One of the interesting facts about the combinatorial theory of convex polytopes is that often algebraic arguments are needed. In some cases one needs a suitable mixture of algebraic and combinatorial arguments. We will see this in various places in this paper.

Remark: Relation 3 also have an algebraic interpretation. Each $r$-face of $P$ determines an $r$-dimensional flat in $R^{d}$ and thus also a vector in the exterior $(r+1)$ power of $R^{d+1}$. The vectors corresponding to all $r$-faces linearly span this exterior power.

\subsection{Polytopes, Simplicial COMPlexes, Simplicial MANifolds, POlyHedral COMPLEXES AND RANKED ATOMIC LATTICES}

The set of faces of a polytope $P$, denoted by $L(P)$ is a ranked atomic lattice. $L(P)$ is called the face lattice of $P$. (A lattice $L$ is ranked if for every element $x \in L$ all maximal chains of elements which are smaller than $x$ have the same size. This size is called the rank of $x$. An atom is an element of rank 1, and $L$ is atomic if every join-irreducible element is an atom.) For example, the face lattice of a simplex is a Boolean lattice. We say that $P$ and $Q$ are combinatorially isomorphic if there is an order preserving bijection between $P$ and $Q$. In most parts of this paper we will not distinguish between combinatorially isomorphic polytopes and we will also by abuse of notation will not distinguish between a polytope $P$ and its face lattice $L(P)$. We say that $Q$ is dual to $P$ and write $Q=P^{*}$, if there is an order reversing bijection between $L(P)$ and $L(Q)$. Every polytope has a dual given by the polar construction (see [24] Ch. 3).

A meet semilattice is a poset with the meet operation. Every finite meet- 
semilattice becomes a lattice by adding to it a maximal element. A polyhedral complex is a meet-semilattice in which every lower interval is combinatorially isomorphic to a face lattice of a polytope. A simplicial complex is a meet-semilattice in which every lower interval is a Boolean lattice. To every polyhedral complex $K$ there is an associated topological space denoted by $|K|$.

Intervals in face lattices of polytopes are also face lattices of polytopes. If $L(Q)$ is combinatorially isomorphic to an interval of $L(P)$ we say that $Q$ is a quotient of $P$. If $F$ is a face of $P$, the interval $[F, P]$ in $L(P)$ is the face lattice of a polytope denoted by $P / F$. For every meet-semilattice $L$ we will use the notation $L / F$ to denote the set of all elements of $L$ which are $\geq$ than $F . L / F$ is called the link of $F$ in $L$. Let $K$ be a simplicial complex and let $F$ be a face of $K$. The star of $F$ in $K$, denoted by $\operatorname{st}(F, K)$ is the simplicial complex spanned by all the faces containing $F$. Note that if $v$ is a vertex of $K$ then $s t(v, K)$ is a cone over the link of $v$ in $F$. Clearly, a link of face in a polyhedral complex is itself a polyhedral complex and a link of a face in a simplicial complex is a simplicial complex.

A simplicial polytope $P$ is a convex polytope all whose (proper) faces are simplices. The set of faces of $P$ is a simplicial complex, denoted by $B(P)$, and called the boundary complex of $P$. If $P$ is a simplicial polytope and $F$ is a face of $P$ then $P / F$ is also a simplicial polytope. The boundary complex of $P / F$ is the link of the face $F$ in the boundary complex of $P$. A simplicial d-sphere is a simplicial complex $K$ such that $|K|$ is homeomorphic to the $d$-dimensional sphere $S^{d}$. Clearly the boundary complex of every simplicial $d$-polytope is a simplicial $(d-1)$-sphere, but the converse is far from being true. However many results on simplicial polytopes extend (or are believed to extend) to arbitrary simplicial spheres.

A ranked atomic lattice $L$ is relatively complemented if every interval in $L$ is atomic. It is sufficient to require that every interval of rank 2 is atomic or, in other words, that if $x>y$ are elements in $L$ and $x$ does not cover $y$, then there are at least 2 elements of $L$ strictly between $x$ and $y$. (See [13].) Clearly, the face lattice of every polytope is relatively complemented.

\subsection{EMPTy FACES}

Let $K$ be a simplicial complex. An empty simplex $S$ of $K$ is a minimal non-face of $K$, i.e., $S$ is a subset of the vertices of $K, S \notin K$ but every proper subset of $S$ is in $K$. Empty simplices are called in $[6,41]$ missing faces. We want to distinguish between empty faces of various types and therefore we use a slightly different terminology.

Lemma 1.4 The set of empty simplices of a simplicial complex $K$ determine the complex.

Proof: A set of vertices of $K$ is a face if and only if it does not contain an empty simplex.

Problem 1 Let $m_{i}(K)$ denotes the number of empty simplices of $K$ of size $i+1$. Characterize the vectors $\left(m_{1}(K), m_{2}(K), \cdots m_{d}(K)\right)$ which arise from simplicial $d$ polytopes.

Let $K$ be a polyhedral complex and let $U$ be a subset of its vertices. The induced subcomplex of $K$ on $U$, denoted by $K[U]$, is the set of all faces in $K$ whose vertices 
are in $U$. An empty face of $K$ is an induced polyhedral subcomplex of $K$ which is homeomorphic to a polyhedral sphere. An empty 2-dimensional face is called an empty polygon.

For the proof of Perles' theorem we need only a much simpler concept of empty pyramid. An empty pyramid of $K$ is an induced subcomplex of $K$ which consists of all the proper faces of a pyramid over a face of $K$.

\section{5. $h$-VECTORS $g$-VECTORS AND THE $g$-THEOREM}

Let $d>0$ be a fixed integer. Given a sequence $f=\left(f_{0}, f_{1}, \ldots, f_{d-1}\right)$ of nonnegative integers, put $f_{-1}=1$ and define $h[f]=\left(h_{0}, h_{1}, \ldots, h_{d}\right)$ by the relation

$$
\sum_{k=0}^{d} h_{k} x^{d-k}=\sum_{k=0}^{d} f_{k-1}(x-1)^{d-k} .
$$

If $f=f(K)$ is the $f$-vector of a $(d-1)$-dimensional simplicial complex $K$ then $h[f]=h(K)$ is called the $h$-vector of $K$. For the case where $K$ is the boundary complex of a simplicial sphere, the $g$-vector $g(K)=\left(g_{0}, g_{1}, \ldots, g_{[d / 2]}\right)$ associated with $K$ is defined by $g_{i}=h_{i}-h_{i-1}$. Thus, $g_{0}=1, g_{1}=f_{0}-(d+1), g_{2}=f_{1}-d f_{0}+\left(\begin{array}{c}d+1 \\ 2\end{array}\right)$ and $g_{3}=f_{2}-(d-1) f_{1}+\left(\begin{array}{c}d \\ 2\end{array}\right) f_{0}+\left(\begin{array}{c}d+1 \\ 3\end{array}\right)$ and so on.

In $1970 \mathrm{P}$. McMullen [36] proposed a complete characterization of $f$-vectors of boundary complexes of simplicial $d$-dimensional polytopes. McMullen's conjecture was settled in 1980. L. Billera and C. Lee [12] proved the sufficiency part of the conjecture and R. Stanley [43] proved the necessity part. Stanley's proof relies on deep algebraic machinery including the hard Lefschetz theorem for toric varieties. Recently, McMullen [37] found a self-contained proof of the necessity part of the $g$ theorem. It is conjectured that the $g$-theorem applies to arbitrary simplicial spheres.

For positive integers $n \geq k>0$ there is a unique expression of $n$ of the form

$$
n=\left(\begin{array}{c}
a_{k} \\
k
\end{array}\right)+\left(\begin{array}{c}
a_{k-1} \\
k-1
\end{array}\right)+\ldots+\left(\begin{array}{c}
a_{i} \\
i
\end{array}\right)
$$

where $a_{k}>a_{k-1}>\ldots>a_{i} \geq i>0$. This given, define

$$
\partial^{k}(n)=\left(\begin{array}{c}
a_{k-1}-1 \\
k-1
\end{array}\right)+\left(\begin{array}{c}
a_{k-1}-1 \\
k-2
\end{array}\right)+\ldots+\left(\begin{array}{c}
a_{i}-1 \\
i-1
\end{array}\right) .
$$

Theorem 1.5 ( $g$-theorem) For a vector $h=\left(h_{0}, h_{1}, \ldots, h_{d}\right)$ of nonnegative integers the following conditions are equivalent:

(i) $h$ is the h-vector of some simplicial d-polytope.

(ii) $h$ satisfies the following conditions
(a) $h_{k}=h_{d-k}$ for $k=0,1, \ldots,\left[\frac{d}{2}\right]$
Put $g_{k}=h_{k}-h_{k-1}$.
(b) $g_{0}=1$ and $g_{k} \geq 0, k=1,2, \ldots,\left[\frac{d}{2}\right]$.
(c) $\partial^{k}\left(g_{k+1}\right) \leq g_{k}, k<\left[\frac{d}{2}\right]$

The relations of part $i$ (a) are the well-known Dehn-Sommerville relations. They hold for arbitrary simplicial spheres and even for arbitrary Eulerian simplicial complexes [35, 3]. Part $i i(b)$ consists of linear inequalities called the generalized lower bound inequalities proposed by McMullen and Walkup in [38]. 
Both Stanley's original proof and McMullen's new proof of the necessity of the $g$-theorem give a (completely different) proof of the same algebraic statement. This algebraic statement can be expresses in terms of the Stanley-Reisner ring [42], or as a property of certain stresses on simplicial polytopes (and also in terms of algebraic shifting [33]). For a thorough explanation the reader is referred to Carl Lee's paper in this volume [2].

The $g$-theorem demonstrates the importance of the $g$-numbers to the combinatorial theory of simplicial polytopes (and spheres). It is natural to ask how combinatorial properties of $P$ are reflected by its $g$-numbers.

A far reaching extension of the $h$-vector (and $g$-vector) for general polytopes was given by Stanley [46], see [1, 29]. We will discuss this in Section 4 .

The following conjecture was suggested by Kalai, Kleinschmidt and Lee. If true, this conjecture gives a sharp form of Theorems 2.7 and 3.8 below.

Conjecture 2 For all simplicial d-polytopes with prescribed $h$-vector $h=\left(h_{0}, h_{1}, \ldots, h_{d}\right)$, the number of $i$-dimensional missing simplices is maximized by the Billera-Lee polytopes $P_{B L}(h)$.

$B P(h)$ is the polytope constructed by Billera and Lee [12] in their proof of the sufficiency part of the $g$-theorem. It is quite possible that the conjecture applies also to general polytopes.

\section{Polytopes with few vertices and Perles' skeleton theorem}

\subsection{Monotonicity PROPERTIES OF $g_{1}$}

Lemma 2.1 For every face $F$ of a polytope $P, g_{1}(P / F)+g_{1}(F) \leq g_{1}(P)$. In particular, $g_{1}(F) \leq g_{1}(P)$ and $g_{1}(P / F) \leq g_{1}(P)$.

Proof: Let $k=\operatorname{dim} F$ and note that $\operatorname{dim}(P / F)=d-k-1 . f_{0}(P / F)$ is the number of $(k+1)$-faces $G$ which contain $F$. Choose a vertex $v_{G}$ in $G$ which is not a vertex of $F$. Clearly if $H$ and $G$ are two different $(k+1)$-faces which contain $F$ then $v_{G} \neq v_{H}$. Therefore $f_{0}(P / F) \leq f_{0}(P)-f_{0}(F)$. So $g_{1}(F)+g_{1}(P / F)=$ $f_{0}(F)-k-1+f_{0}(P / F)-(d-k) \leq f_{0}(P)-d-1=g_{1}(P)$.

Lemma 2.2 [10] Put $\nu(P)=\operatorname{Max}\left\{f_{0}(P)-f_{0}(F)-1: F\right.$ is a facet of $\left.P\right\}$. Then for every facet $F$ of $P, \nu(P) \geq \nu(F)$.

Proof: Let $F$ be a facet of $P$ and let $G$ be a facet of $F$. $G$ is the intersection of $F$ with another facet $F^{\prime}$ of $P$. Thus $V(P) \backslash V\left(F^{\prime}\right) \supset V(F) \backslash V(G)$. Therefore $f_{0}(P)-f_{0}\left(F^{\prime}\right) \geq f_{0}(F)-f_{0}(G)$. The Lemma follows.

Note that $\nu(P)=0$ if and only if $P$ is a simplex.

Lemma 2.3 Every $d$-polytope with $d+b$-vertices contain $a(d-b+1)$-face which is a simplex.

Proof: Choose a sequence $P \supset F_{d-1} \supset F_{d-1} \supset \cdots \supset F_{d-b}=G$ such that $\nu\left(F_{i}\right)=f_{0}\left(F_{i}\right)-f_{0}\left(F_{i-1}\right)-1$. If $G$ is not a simplex then $\left.\nu(G)>0\right)$ and therefore by the previous Lemma $\nu\left(F_{i}\right)>0$ for every $i>d-b+1$. Therefore $g_{1}(P) \geq$ $g_{1}(G)+b-1 \geq b$. A contradiction. 


\subsection{The simplicial CASE of Perles' theorem}

Definition: A collection $\left\{A_{1}, A_{2}, \cdots, A_{t}\right\}$ of sets is a sunflower if every element which belongs to two or more of the sets belongs to all the sets. In other words, let $X=\cap_{i=1}^{t} A_{i}$ then for every $i \neq j A_{i} \cap A_{j}=X$.

Lemma 2.4 Let $P$ be a simplicial d-polytope, and assume that $P$ contains $b$ disjoint empty simplices, then $P$ has at least $d+b$ vertices.

Proof: Let $F$ be a facet of $P$, clearly every empty simplex of $P$ must contain a vertex not in $F$, but $V(P) \backslash V(F)=f_{0}(P)-d=b$. (Here $V(F)$ denotes the set of vertices of $F$.)

Lemma 2.5 Let $P$ be a simplicial d-polytope, and assume that $P$ contains a sunflower of size $b$ of empty simplices, then $P$ has at least $d+b$ vertices.

Proof: First note that if $S$ is an empty simplex in $P$ and $A \subset S$ then $S \backslash A$ is an empty simplex in $P / A$. Now, if $P$ contain a sunflower $\left\{A_{1}, A_{2}, \cdots A_{b}\right\}$ of empty simplices and $\cap_{i=1}^{b} A_{i}=R$ then $A_{1} \backslash R, A_{2} \backslash R, \ldots, A_{b} \backslash R$, are $b$ disjoint empty simplices in $Q=$ $P / R$. Therefore by Lemma $2.5 f_{0}(Q)-\operatorname{dim} Q \geq b$ and by Lemma $2.1 f_{0}(P)-d \geq b$.

Lemma 2.6 (Erdős-Rado sunflower lemma [20]) Let $F$ be a collection of $n$ sets which contains no sunflower of size $b$ then $|F| \leq m(n, b)=(b-1)^{n} \cdot n !$.

Proof By induction on $n$. Let $F$ be a collection of $n$-sets with out a sunflower of size $b$, and let $G$ be a maximal subcollection of pairwise disjoint sets. Put $A=\cup G$. Then $|G|<b,|A| \leq n(b-1)$ and every set in $F$ contains an element from $A$. For each $a \in A$ the family $F(a)=\{S \backslash\{a\}: S \in F, a \in S\}$ is a family of $(n-1)$ sets without a sunflower of size $b$. Using the induction hypothesis we get $|F| \leq$ $n(b-1) \cdot(b-1)^{n-1}(n-1) !=m(n, b)$.

Let me mention an old and still very interesting conjecture of Erdös and Rado. Let $f(n, b)$ be the maximum size of a family of $n$-sets without a sunflower of size $b$.

Conjecture 3 (Erdös and Rado) For a fixed $b f(n, b) \leq C(b)^{n}$, where $C(b)$ is a function of $b$ only.

As a corollary to the results we proved in this section we obtain:

Theorem 2.7 Let $P$ be a d-polytope with $d+b$ vertices. Then the total number of empty simplices of dimension $\leq k$ is bounded by a function of $b$ and $k$.

Proof of Perles' theorem, the simplicial case:

We want to bound the combinatorial types of $k$-skeleta of simplicial $d$-polytopes $P$ with $d+b$ vertice. The $k$-skeleton of $P$ is determined by the set of empty simplices of $P$ of dimension $\leq k$. The number of empty simplices of dimension $\leq k$ is bounded by $m(k+1, b+1)=b^{k+1} \cdot(k+1)$ !. Therefore, the number of all vertices of these empty faces is bounded by $(k+1) \cdot m(k+1, b+1)$ and the number of isomorphism types of the family of empty simplices is at most $\sum_{i=1}^{k+1}\left(\begin{array}{c}(k+1) m(k+1, b+1) \\ i\end{array}\right)$. This is roughly $\exp \left((k+1)^{2}(\log (k+1)+\log b-1)\right.$. 


\subsection{Perles theorem - The General CASE}

Lemma $2.8 A$ d-polytope $P$ with $d+b$ vertices has at most $2 b$ disjoint empty pyramids.

Proof: In follows from Lemma 2.3 that $P$ must have a $(d-b-1)$-face $S$ which is a simplex. Every empty pyramid (or empty face) must contain vertices outside $S$. The lemma follows from the fact that $|V(P) \backslash V(S)|=2 b$.

Lemma 2.9 Every collection of more than $(b-1)^{r} \cdot n^{r} r$-faces each having at most $n$ vertices, contains a sunflower of size $b$.

Proof: The proof follows the inductive proof of the sunflower lemma. Let $F$ be a collection of faces of dimension $r$ (or less) each having $n$ vertices or less without a sunflower of size $b$. Let $G$ be a maximal subcollection of pairwise disjoint faces. Let $A$ be the set of vertices of all faces in $G$. Then $|G|<b,|A| \leq n(b-1)$ and every set in $F$ contains an element from $A$. For each $a \in A$ the family $F(a)=\{S /\{a\}: S \in$ $F, a \in S\}$ is a family of $(r-1)$-faces with at most $(n-1)$ - vertices without a sunflower of size $b$. Using an induction hypothesis we get $|F| \leq n(b-1) \cdot(b-1)^{r-1} n^{r-1}$.

Proof of Perles' theorem (end): ${ }^{1}$ For a polyhedral complex $K$ define the kernel of $P, \operatorname{Ker}(P)$, to be the union of the sets of vertices of all empty pyramids in $K$. Clearly the combinatorial type of $K \operatorname{er}(K)$ and the number of vertices of $K$ determine the combinatorial type of $K$. Namely the set of faces of $K$ is precisely $F * T$ where $F$ is a face of $K$ and $T$ is any subset of vertices which are disjoint from $\operatorname{Ker}(K) . F * T$ is the $|T|$-fold pyramid with basis $F$.

Lemmas 2.9 implies that the kernel of the $k$-th skeleton of a $d$-polytope with $d+b$ vertices has at most $\left.(2 b)^{r-1}(b+k)\right)^{r}$ vertices. Therefore the number of isomorphism types of $k$-skeleta of $d$-polytopes with $(d+b)$ vertices is bounded by the number of $k$-dimensional polyhedral complexes with $\left.(2 b)^{r-1}(b+k)\right)^{r}$ vertices.

\subsection{The SCOPE of Perles' TheOrem}

As easily seen the proof of Perles theorem for simplicial polytopes given above applies for arbitrary pure simplicial complexes. The proof of the general case applies for a large class of ranked atomic lattices. Perles observed that his proof (and this applies to the proof given here) applies to arbitrary ranked atomic relatively complemented lattices. He went further to define an even larger class of lattices, the class of pyramidally perfect lattices, for which his proof applies. For an element $x$ in an atomic lattice $L, J(x)$ denotes the set of atoms below $x$. An atom $a$ is pyramidal with respect to $x \in L$ if $a \nless x$ and $J(x \vee a)=J(x) \cup\{a\}$. A ranked atomic lattice is called pyramidally perfect if whenever $a$ is pyramidal w.r.t. $\mathrm{x}$ it is also pyramidal w.r.t. every $y, y<x$,

\section{Simplicial polytopes with small value of $g_{k}$}

\subsection{OVERVIEW}

In this section we discuss simplicial polytopes with small value of $g_{k}$. The situation is simpler for $g_{2}$ and more involved for higher $k$ 's.

\footnotetext{
1 This part of the proof is taken from [41] without changes
} 
The nonnegativity of $g_{2}$ can be proved by purely combinatorial methods as well as by the rigidity theory of frameworks. Both approaches apply to a very general class of simplicial complexes, the class of pseudomanifolds. The rigidity theoretic interpretation of $g_{2}$ gives much information on the structure of simplicial polytopes (and simplicial manifolds) with small values of $g_{2}$. This is described below in Sections 3.3. The proofs of the necessity of the $g$-theorem (both Stanley's original proof and McMullen recent proof) deduce the theorem from a certain crucial algebraic fact. This gives an interpretation of $g_{k}$ which is closely related to the rigidity theoretic interpretation of $g_{2}$ see $[2,37,33]$ and allows to extend some of the results to simplicial polytopes with small values of $g_{k}$.

In Section 3.2 we state a conjecture giving a complete description of $g$-vectors of sequences of simplicial polytopes which converge to smooth bodies. Like the $g$ theorem the conjecture consists of a linear part and a nonlinear part. The linear part of the conjecture may be doable by improving the methods and results described here. In Section 3.3 we describe the main tool we use, the notion of stresses. This is a very quick outline of some facts from Carl Lee's paper [2]. In Section 3.4 we state the lower bound inequalities and in Section 3.5 we describe the structure of the proof showing that $g_{2}=0$ only for stacked polytopes. In Section 3.6 we describe some partial information on polytopes with vanishing $g_{k}$. In Section 3.7 we extend Perles theorem to simplicial polytopes with bounded $g_{k}$. In Section 3.8 we study in more details the case $k=2$. It turns out that every simplicial polytope with small value of $g_{2}$ can be obtained by gluing together "small" pieces. In Section 3.8 we diverge to describe finer invariants of simplicial polytopes which give much more information than the $g$-numbers.

\section{2. $g$-NUMBERS OF SIMPLICIAL POLYTOPES WHICH CONVERGE TO A SMOOTH BODY}

We state two conjectures on the behavior of $g$-numbers of simplicial polytopes which converge to a smooth body. The first conjecture falls into our study of polytopes with a bounded values of $g_{k}$. It is trivial for $k=1$ and follows from the result of Section 3.8 for $k=2$. The second conjecture calls for a similar study of polytopes for which $g_{k}^{<k>}-g_{k+1}$ is bounded.

Conjecture 4 ([26]) Let $k, d$ be positive integers $d \geq 2 k$. Let $P_{n}$ be a sequence of $d$-polytopes which converge to a smooth body $K$. Then

$$
\lim _{n \rightarrow \infty} g_{k}\left(P_{n}\right) \rightarrow \infty
$$

Conjecture 5 Let $P_{n}$ be a sequence of d-polytopes which converge to a smooth body $K$. Then for $k<[d / 2]$

$$
\lim _{n \rightarrow \infty}\left(g_{k}\left(P_{n}\right)-\partial^{k}\left(g_{k+1}\left(P_{n}\right)\right)\right) \rightarrow \infty
$$

If Conjectures 4 and 5 are true then they give a complete descriptions of sequences of $g$-vectors which come from sequence of simplicial polytopes converging to a smooth body $K$. (Note: the description is independent from $K$.)

If $P_{n}$ is a sequence of polytopes which converges to a convex body $K$, and $Q_{n}$ is any sequence of polytopes, then one can glue a projective copy of $Q_{n}$ to one facet of $P_{n}$ and the resulting sequence of polytopes will also converges to $K$. 
If $\mathcal{G}_{d}$ is the set of $g$-vectors of simplicial $d$-polytopes, and $B_{d}(r)$ is the set of vectors $\left(a_{1}, \ldots, a_{[d / 2]}\right)$ such that $\left|a_{i}\right| \leq r$ for every $i$. Then if Conjectures 4 and 5 are true, a sequence of $g$-vectors $\left\{g^{n}\right\}$ is the sequence of $g$-vectors of simplicial polytopes converging to a smooth convex body $K$ if and only if for every $r$ there is $N_{r}$ such that $g^{n}+B_{d}(r) \subset \mathcal{G}_{d}$ for every $n>N_{r}$.

Remarks: 1. Connections between metrics on the sphere and combinatorial invariants

It is possible to formulate similar questions in a purely combinatorial way for sequences of simplicial spheres (and even simplicial manifolds). Every triangulation of the sphere induces a metric and it is possible to consider limits of such metrics (see [23]) as the number of vertices tends to infinity. It is natural to study Conjectures 4 and 5 in this more general context, and more generally to study the following

Problem 6 Given a metric on the $(d-1)$-sphere and sequence of simplicial spheres whose induced metrics converges to this metric, find relations between the $g$-numbers (and other combinatorial properties) of the simplicial spheres in the sequence and the geometric properties of the limiting metric.

2. Separation properties of $G\left(P^{*}\right)$.

Let $K$ be a pure $(d-1)$-dimensional simplicial complex. The dual graph of $K$, denoted by $G^{*}(K)$ is defined as follows. the vertices of $G^{*}(K)$ are the facets (= $(d-1)$-faces $)$ of $K$ and two vertices $F$ and $G$ are adjacent if $F \cap G$ is a $(d-2)$ dimensional face. If $K$ is the boundary complex of a simplicial polytope $P$ then $G^{*}(K)$ is just the graph of the dual polytope $P^{*}$.

Let $f(r)$ be a function of the integer number $r$ and consider the class $\mathcal{P}_{f}$ of all simplicial polytope $P$ (or even simplicial spheres) with the following property. Whenever $r$ vertices are deleted from $G\left(P_{n}^{*}\right)$ then the remaining graph has a connected component having all vertices accept at most $f(r)$ of them. It is plausible (and would imply Conjecture 4) that for every function $f(r)$ and for all polytopes in $\mathcal{P}_{f}$ the value of $g_{k}(P)(k<[d / 2])$ is bounded. See [33] for some results on separation properties of graphs of special types of simple polytops.

\subsection{STRESSES}

This is a very quick outline of some important ingredients of Carl Lee's paper [2]. See also the papers of Tay, White and Whiteley [50] on skeletal rigidity. The reader should also consult Lee's paper for the relations between stresses and the StanleyReisner's ring and [33] for the connection with algebraic shifting.

Let $K$ be a simplicial complex embedded into $R^{d}$. (By "embedded" we only mean that the vertices are embedded in such a way that the vertices of faces are affinely independent.) A $k$-stress (which is an abbreviation here for an affine $k$-stress since we will not consider linear stresses, ) is defined as follows. For every $(k-1)$-face $F$ choose a point $u_{F} \in F$. If $G$ is a $k$-face containing a $(k-1)$-face $F$ let $v(F, G)$ denotes the vertex of $G$ not in $F$. A $k$-stress is an assignment of weights $w_{G}$ to the $k$-faces $G$ of $K$ such that for every $(k-1)$-face $F$

$$
\sum\left\{w_{G}\left(v(F, G)-u_{F}\right): G \supset F\right\} \in A f f(F) .
$$


Here, $\operatorname{Aff}(F)$ is the affine span of the face $F$. Let $S_{k}^{a}$ denotes the space of $k$ stresses of $K$.

Let $A_{k}(K)$ be the space of all assignment of weights $w_{G}$ to the $k$-faces $G$ of $K$. Now consider the map $T_{k}$ which assigns to every $w \in A_{k}(K)$ weights on $(k-1)$-faces $F$ as follows: The weight of a $(k-1)$-face $F$ is $\sum\left\{w_{G}\left(v(F, G)-u_{F}\right): G \supset F\right\}$ considered as a vector in the quotient space $R^{d} / A f f(F)$. (The weights are vectors of dimensions $d-k+1$.) The space of $k$-stress is precisely the kernel of $T_{k}$. There is a complementary notion of $k$-rigidity, (or skeletal $k$-rigidity) which is of importance here. Roughly speaking, $K$ is $k$-rigid if the image of $T_{k}$ is "as large as possible". What we will need is that if $K$ is $k$-rigid, and $K^{\prime}$ is obtained from $K$ by adding just one $k$-face then $K^{\prime}$ has a non-zero $k$-stress.

All these concepts become classic for $k=1$. 1-rigidity is called infinitesimal rigidity. 1-stress is the classical notion of a stress of a framework. 0-stresses are just affine relations among the vertices, and being 0-rigid just mean that the vertices of $K$ affinly span $R^{d}$

The basic fact connecting stresses with the $g$-vector is the following fact which follows from the two known proofs of the necessity part of the $g$-theorem. (In Stanley's proof the following fact is proved only for rational polytopes.)

Theorem 3.1 Let $P$ be a simplicial d-polytope let $k<[(d+1) / 2]$. Then $g_{k}(P)=$ $\operatorname{dim} S_{k}^{a}(P)$.

This is equivalent to the fact that $\operatorname{Skel}_{k}(P)$ is $k$-rigid.

An important fact about stresses is that they behave nicely under forming a cone. Let $K$ be a simplicial $k$-dimensional complex and consider a generic embedding of $K$ in $R^{d}$. Consider also a generic embedding of a cone over $K$ in $R^{d+1}$. Then the embedding of $K$ is $k$-stress free iff the embedding of the cone is $k$-stress free. See [50]. (This is related to the fact that the operation of forming a cone commutes with algebraic shifting.)

Remark: Stresses can be regarded as analogs for Gale transforms which are one of the most useful tools in the study of polytopes with few vertices. However, the extension of the basic property of Gale transform is not yet known:

Conjecture 7 ([26]) Let $P_{1}$ and $P_{2}$ be two simplicial d-polytopes and let $\phi$ be $a$ bijection from $V(P)$ to $V(Q)$ such that, $\phi$ is a combinatorial isomorphism from $\operatorname{Skel}_{k}(P)$ to $\operatorname{Skel}_{k}(Q)$ and moreover the map induced by $\phi$ gives an isomorphism between the space of $k$-stresses of $P$ and the space of $k$-stresses of $Q$. Then $\phi$ induces a combinatorial isomorphism between $P$ and $Q$.

In other words, is the $k$-skeleton plus the vector space of $k$-stresses determine uniquely the combinatorial type of simplicial polytopes?

Note that for $k=[d / 2]$ the space of stresses is trivial, but indeed an important theorem of Perles asserts that for two simplicial $d$-polytopes every combinatorial isomorphism between $\operatorname{Skel}_{[d / 2]}(P)$ and $S k e l_{[d / 2]}(Q)$ can be extended to a combinatorial isomorphism between $P$ and $Q$. Also, as we shall see later, if for $k<[d / 2]$ the space of $k$-stresses of a simplicial $d$-polytope is trivial (i.e. $g_{k}(P)=0$ ) then $P$ has no missing faces in dimensions greater than $k$ and smaller then $d-k$ and again the $k$-skeleta determine the combinatorial structure of the polytope. 


\section{4. $g_{2}$ AND THE LOWER BOUND INEQUALITIES}

A simplicial $d$-polytope is stacked if it can be obtained by gluing $d$-simplices along facets. Every stacked polytope with $n$ vertices is obtained from a stacked polytope with $n-1$ vertices by adding a vertex beyond exactly one facet. $P$ is stacked if and only if it can be triangulated without introducing faces of dimension smaller than $(d-1)$. The boundary complex of a stacked polytope is called a stacked sphere.

It is easy to see that the $f$-vector of a stacked $d$-polytopes is determined by the number of vertices. Let $\phi_{k}(n, d)$ denotes the number of $k$-faces of a stacked $d$-polytope with $n$ vertices. Thus, $\phi_{k}(n, d)=\left(\begin{array}{l}d \\ k\end{array}\right) n-\left(\begin{array}{l}d+1 \\ k+1\end{array}\right) k$, for $k, 1 \leq k \leq d-2$, and $\phi_{d-1}(n, d)=(d-1) n-(d+1)(d-2)$.

The lower bound inequalities assert that for every simplicial $d$-polytope $P$ with $n$ vertices, $f_{k}(P) \geq \phi_{k}(n, d)$. The case $k=1$ of this inequality is just the nonnegativity of $g_{2}$. There is an inductive way to deduce the lower bound inequalities from the nonnegativity of $g_{2}$. See $[7,38,27]$. However, this inductive argument does not apply for certain generalization of the lower bound inequalities such as for centrally symmetric polytopes and for general polytopes. Thus, it may be useful to find a direct interpretation of $g_{2}[r]=f_{k}(P)-\phi_{k}(n, d)$ as the dimension of some vector space.

\subsection{Simplicial POLYTOPE With VANiShing $g_{2}$}

Theorem 3.2 For $d>4$ the following conditions are equivalent: (1) $P$ is stacked (2) $P /\{v\}$ is stacked for every vertex $v$, (3) $P$ has no empty simplices of dimension $r$, for $1<r<d-1$.

The proof of this theorem is given in [27]. It applies to arbitrary simplicial $(d-1)$-manifolds which are simply connected. For non-simply connected manifolds $M$ conditions 2 and 3 remain valid and are equivalent to the fact that $M$ is obtained from the boundary of stacked polytope by additional operations of handle forming via identifying the vertices of two disoint facets and deleting the facet.

Theorem 3.3 For $d \geq 4$ the following are equivalent (1) $P$ is stacked (2) $P$ has no empty faces (of any kind) of dimension $r$, for $1<r<d-1$ (3) $P$ has no empty simplices of dimension $r$, for $1<r<d-1$ and no empty polygons.

The crucial point behind this theorem is the situation for simplicial 3-polytope. A simplicial 3-polytope is stacked iff it has no missing polygons other than triangles. While the two theorems above are purely combinatorial rigidity arguments are needed to prove the following

Theorem 3.4 For $d>3$, if $g_{2}=0$ then

(1) $P$ has no empty faces of dimension $r, 1<r<d-1$ (2) $g_{2}(P / v)=0$ for every vertex $v$. (3) $P$ has no empty polygons.

It follows from the theorems quoted above that if $g_{2}(P)=0$ then $P$ is a stacked polytope. This result applies to arbitrary simplicial manifolds (and pseudomanifolds). 
Remark: There is an interesting issue which is related to the above theorems. Consider a simplicial manifold $K$ and assume that all links of vertices $K / v$ (which are simplicial spheres) have certain combinatorial properties. What does this imply on the topology of $K$ ? If all links are stacked spheres then for dimensions $>3$ this implies severe restrictions on the topology of $K$, and in particular, if $K$ is simply connected then $K$ is a sphere (the 3 -dimensional case is open).

\subsection{Simplicial polytopes With VANishing $g_{k}$}

We do not have good understanding of simplicial polytopes such that $g_{k}(P)=0$. McMullen and Walkup conjectured that every such polytope is $k$-stacked, namely it can be triangulated without introducing faces of dimensions $<d-k$. Unlike stacked (1-stacked) polytopes which are well understood, $k$-stacked polytopes are themselves quite mysterious. Parts of the discussion concerning the vanishing of $g_{2}$ extends to higher $k$ 's but other parts are still not known (but perhaps doable).

Proposition 3.5 For $d \geq 2 k+3$ the following are equivalent (1) $P$ is $k$-stacked (2) $P / v$ is $k$-stacked for every vertex $v$.

Proposition 3.6 For $d>2 k+1$, if $g_{k}(P)=0$ then (1) $P$ has no empty simplexs of dimension $r, k \leq r \leq d-k$ (2) $g_{k}(P / v)=0$ for every vertex $v$.

Proof: (1) Assume that $S$ is an empty $k$-simplex. Now, the vertex figure $P / v$ is $k$-rigid and therefore $s t(v, P)$ (being a cone over it) is $k$-rigid. $R=S \backslash v$ is a $(k-1)$ face in $P$ which is not in $s t(v, P)$. Therefore $s t(v, P) \cup R$ has a non-zero stress and since $s t(v, P) \cup R \subset P, P$ has a nonzero $k$-stress, and $g_{k}(P)>0$. If $S$ is an empty simplex of size $k+i$ choose $V \subset S,|V|=i$ and a vertex $v \in V$. Apply the same argument for $P / V$ inside $P /(V \backslash\{v\})$.

Part (2) follows at once from the cone property for $k$-stresses. In fact, we get

Lemma $3.7 g_{k}(P / v) \leq g_{k}(P)$.

Proof: $g_{k}(P / v)$ is the dimension of the space of $k$-stresses of $P / v$ w.r.t to embedding in $R^{d-1}$, therefore $g_{k}(P / v)$ is the dimension of the space of $k$-stresses of $s t(v, P)$ w.r.t. embedding in $R^{d}$.

Part (2) of Proposition 3.6 also follows from the identity

$$
\sum_{v} g_{k}(P / v)=(d-k+1) g_{k}(P)+(k+1) g_{k+1}(P),
$$

and the nonnegativity of $g_{k+1}(P)$.

Conjecture 8 For $d \geq 2 k$ the following are equivalent (1) $P$ is $k$-stacked (2) $P$ has no empty faces (of any kind) of dimension $r$, for $k<r<d-k$ for $d \geq 2 k$ these two conditions are equivalent to (3) $g_{k}(P)=0$.

Remark: The $k$-the skeleton of every $d$-polytope contains a subdivision of the $k$-skeleton of a $d$-simplex. For simplicial polytopes nonvanishing of $g_{k}$ also seems related to the existence of a subdivision of the $k$-skeleton of a $(d+1)$-simplex. Indeed, nonvanishing of $g_{2}$ for a simplicial polytope $P$ is equivalent to the fact that the graph of $P$ contains a refinement of $K_{5}$ [27]. For $k>2$ results of Stanley [48] seems relevant. 


\subsection{Analog of Perles theorem for simplicial polytopes With small VALUE OF $g_{k}$}

Theorem 3.8 For positive integers $k \geq 1, r \geq k$ and $b \geq 0$ there is a function $b_{k}(r, b)$ with the following property: Let $P$ be a simplicial d-polytope with $g_{k}(P) \leq b$. Then $P$ has at most $b_{k}(r, b)$ empty $r$-simplices.

\section{Proof (sketch)}

By the sunflower theorem it is enough to prove that there is no sunflower of empty $r$-simplices of size $b+1$. We have seen that an empty $r$-simplex, $d-k>r>k$ is responsible to a $k$-stress, so it is enough to show that in case of a sunflower $\left\{S_{1}, S_{2}, \ldots, S_{b+1}\right\}$ we get a contribution of at least $b+1$ to the space of stresses. Let $R$ be the intersection of the $S_{i}$ 's. If $|R|>r-k$ the situation is very easy; choose $V \subset R$ such that $|V|=r-k$ and a vertex $v \in V$. Let $K=P /(V \backslash\{v\}) . K / v$ is $k$-rigid in dimension $d-|V|$ and therefore $s t(v, K)$ is $k$-rigid in dimension $d-|V|+1$ and adding to it the $b+1 k$-faces $S_{1} \backslash V, S_{2} \backslash V \ldots, S_{b+1} \backslash V$ create a $k$-stress space of dimension at least $b+1$. The case where $|R|$ is smaller is slightly more complicated and we omit the details.

Corollary 3.9 There exists a function $u_{k}(r, b)$ with the following property: Let $K$ be the $k$-th skeleton of a simplicial d-polytope $P$ with $g_{k}(P)<b$ (Note: $g_{k}$ can be read from the $k$-skeleton.) Then there are only $u_{k}(r, b)$ possibilities for the $r$-skeleton of $P$.

Note that the number of 1 -skeleta of stacked $d$-polytopes with $n$ vertice is exponential in $n$.

It seems that Theorem 3.8 applies to general empty faces and not only to empty simplices. What we need to do is given an empty face in dimension $r, k<r<$ $d-k$ to find a nonzero $k$-stress, such that for disjoint empty faces one gets linearly independent $k$-stresses. It looks that an appropriate Meyer-Vietoris type statement for $k$-stresses is needed. Proving this may be helpful also in verifying Conjecture 4 .

\subsection{Simplicial POLYTOPES With SMALL VALUE OF $g_{2}$}

A simplicial polytope $P$ is prime if it does not contain an empty $(d-1)$-simplex. If $P$ is not prime then $P$ can be obtained by gluing together along facets of prime simplicial polytopes. We write $P=P_{1} \# P_{2} \# \cdots \# P_{k}$ for the description of $P$ as the union of prime simplicial polytopes. It is easy to see that $g_{2}(P)=\sum g_{2}\left(P_{i}\right)$. The following theorem shows that if $g_{2}(P)$ is small then $P$ is obtained by gluing together many small pieces. (Clearly most of these pieces must be simplices.)

Theorem 3.10 There is a function $u(d, b)$ such that if $P$ is a prime simplicial $d$ polytope and $g_{2}(P) \leq b$ then $g_{1}(P) \leq u(d, b)$.

Proof: (sketch) For $d>4$. We know that the number of empty simplices of $P$ of dimension $r, 1<r<d-1$ is at most $X=X(d, b)$. The number of edges of $P$ is bounded by a linear function of the number of vertices. Therefore (by Turán theorem) if the number of vertices is large then $G(P)$ contains an independent set $U$ of vertices of size $>X$. For $u \in U$, if $P /\{u\}(=$ the link of $u$ in $P$ ) is not stacked 
and also if $P /\{u\}$ contains a empty $d-2$ simplex which is not a face of $P$, we get a non-zero stress $s_{u}$ is $s t(u, K)$. Moreover $s_{u}$ have nonzero weight on some edge containing $u$. Since $U$ is an independent set of vertices this edge is not included in the star of any other vertex of $U$. Therefore, all the stresses $\left\{s_{u}: u \in U\right\}$ are linearly independent. A contradiction.

It follows that for some $u \in U, \operatorname{st}(u, P)$ does not contain a non-zero stress. Therefore either $P /\{u\}$ is a simplex and thus an empty $(d-1)$-simplex or $P /\{u\}$ is stacked, and contains an empty $(d-2)$-simplex $R$, such that $R$ is not an empty simplex in $P$. In this case $R \cup\{u\}$ is an empty $(d-1)$-simplex in $P$. The proof for $d=4$ is more involved and we will not include it here.

Remarks: 1 . It is quite possible that $u(d, b)$ is actually independent from $d$, and it would be interesting to determine its best possible value. I do not know of examples of prime simplicial polytopes with $g_{1} \leq g_{2}-1$.

2. The proof of Theorem 3.10 applies in much more general contexts. The proof as described above applies for simplicial spheres and even for simplicial manifolds. Every simply connected prime simplicial manifold can written as a connected sum of prime (simply connected) simplicial manifolds. For arbitrary manifolds one has to add another operation - that of "handle forming" via an identification of two facets. Every simplicial manifold with small value of $g_{2}$ can be obtained from small "prime" pieces by the operations of connected sum and handle forming. (Every handle increase the value of $g_{2}$ by $\left(\begin{array}{c}d+1 \\ 2\end{array}\right)$.) It follows that for every $b$ there are only finitely many $d$-manifolds which have a triangulation $K$ such that $g_{2}(K) \leq b$.

3. Even more generally, Theorem 3.10 applies to all pseudomanifolds such that the link of every face of codimension $\geq 1$ is connected and the link of every link of dimension $\geq 2$ is simply connected.

\subsection{Diversion: Finer invariants}

An order ideal of monomials $I$ is a collection of monomials in variables (say) $x_{1}, x_{2}, \ldots$ such that $1 \in I$ and if $m \in I$ and $m^{\prime}$ divides $m$ then $m^{\prime} \in I$. We will denote by $f_{k}(I)$ the number of monomials of $I$ of degree $k$ and call the vector $\left(f_{0}(I), f_{1}(I), \ldots\right)$ the $f$-vector of $I$. (Note: the indices here are shifted by 1.) An order ideal of monomial is shifted if for every monomial $m$ in $S$ if $x_{j}$ has positive degree in $m$ and $i<j$ then $m \cdot x_{i} \cdot x_{j}^{-1} \in I$.

A sequence of integers $\left(m_{0}, m_{1}, \ldots\right)$ is an M-vector if $m_{0}=1$ and $0 \leq \partial^{k}\left(m_{k+1}\right) \leq$ $m_{k}$ for every $k \geq 1$. An old theorem of Macaulay asserts that M-vectors are precisely the possible $f$-vectors of order ideal of monomials. Every M-vector can be realized (usually in many ways) as the $f$-vector of a shifted order ideal of monomials.

For a simplicial $d$-polytope (and probably for every simplicial $(d-1)$-sphere,) the vector $\left(1, g_{1}, \ldots, g_{\left[\frac{d}{2}\right]}\right)$ is an $M$ - vector. The proof of the necessity part of the $g$-theorem actually associates to every simplicial polytope $P$ a shifted order ideal of monomials $S(P)$ such that $g_{i}(K)$ is the number of monomials of degree $i$ in $S$. (See [33].) $S(P)$ can be regarded as a delicate invariant of $P$. It is conjectured that the same algebraic construction applies to arbitrary simplicial spheres.

Conversly, for every shifted order ideal of monomial $S$ there is a construction of a simplicial sphere $K(S)$, see [28]. It is conceivable but not known that $S(K(S))=S$.

It would be interesting to study the structural properties of $P$ as a function of 
$S(P)$, in a similar line to the approach of this paper. Here is a far-reaching extensions of Conjectures 4 and 2 .

Let $M(d)$ denotes the set of all monomials of degree $\leq[d / 2]$ on the countable set of variables $x_{1}, x_{2}, \ldots, x_{n}, \ldots$.

Conjecture 9 Let $P_{n}$ be a sequence of simplicial d-polytopes which converges to a smooth body $K$. Then $\cup S\left(P_{n}\right)=M(d)$.

Conjecture 10 For simplicial spheres $K$ with $S(K)=S$, the vector of empty simplices is maximizes for the complex $K(S)$.

Remarks: 1. It is known for quite a long time that there are simplicial (and polyhedral) spheres that cannot be realized as the boundary complex of a simplicial polytope. In high dimensions there is a striking gap between the number of combinatorial types of simplicial polytopes and the number of combinatorial types of simplicial spheres. See [22, 28]. However, most of the results mentioned here for simplicial polytopes are either known or conjectured to be known for simplicial spheres. While face numbers are probably too weak to distinguish simplicial polytopes from arbitrary triangulations of spheres it is possible that the finer invariant $S(K)$ will contain some useful parameters for this problem.

2. It is interesting to note that neither the $g$-vectors nor the finer invariant $S(P)$ can distinguish between different neighborly polytopes. Indeed $P$ is neighborly iff $S(P)$ is the ideal of all monomials of degree $\leq d / 2$ in $n-d$ variables. The combinatorial structure of neighborly polytopes (even in dimension 4 ) is a rich topic and it seems that completely different invariants are needed for their study.

\section{General Polytopes}

\subsection{OVERVIEW}

In this section we consider general polytopes. In this case even the definition of $g_{k}(P)$ is quite subtle. We describe the definition in Section 4.2. More details can be found in Bayer's paper [1]. Section 4.3 is devoted to $g_{2}(P)$. We describe the rigidity theoretic meaning of $g_{2}(P)$, and describe some facts on the remarkable class of polytopes with vanishing $g_{2}$. The nonnegativity of the $g_{k}$ 's implies many linear inequalities for flag numbers of polytopes. The possibility to use the large amount of complicated data given by such inequalities to prove basic and easy to state properties of polytopes is discussed in Section 4.4. We describe there results of Meisinger who developed an automatic polytope theorem prover FLAGTOOL. In Section 4.5 we make some conjectures for additional linear inequalities for flag numbers of polytopes. In Section 4.6 we discuss special classes of polytopes and in Section 4.7 we ask to which generality can we hope for a notion of $h$ - and $g$-numbers.

\section{2. $g_{k}$ FOR GENERAL POLYTOPES AND FLAG NUMBERS}

Intersection homology theory have led to deep and mysterious extensions of $g$ numbers from simplicial polytopes to general polytopes.

The definition (which can be found also in $[1,3]$ ) goes as follows. For a polytope $P$ denote by $P_{k}$ the set of $k$-faces of $P$. 
Define by induction two polynomials

$$
h_{P}(x)=\sum_{k=0}^{d} h_{k} x^{d-k}, g_{P}(x)=\sum_{k=0}^{[d / 2]} g_{k} x^{d-k}
$$

by the rules: (a) $g_{k}=h_{k}-h_{k-1}$, (b) If $P$ is the empty polytope or a 0 -polytope $P$, $h_{P}=g_{P}=1$, and

$$
h_{P}(x)=\sum_{k=0}^{d}(x-1)^{d-k} \sum\left\{g_{F}(x): x \in P_{k}\right\} .
$$

Thus $g_{1}(P)=f_{0}(P)-d-1$ and

$$
g_{2}(P)=f_{1}(P)+\sum\left\{f_{0}(F)-3: F \in P_{2}\right\}-d f_{0}(P)+\left(\begin{array}{c}
d+1 \\
2
\end{array}\right) .
$$

The value of $g_{2}$ for general polytopes have also a rigidity theoretic meaning. In this case, however, the nonnegativity of $g_{2}$ is still open for more general objects like polyhedral spheres and manifolds.

The higher $g$ numbers for general polytopes are quite mysterious, and at present their nonnegativity is known only for polytopes with rational vertices. Goresky and MacPherson (unpublished) developed a concrete way to describe $g_{k}(P)$ as certain hyperhomology groups based directly on the geometry of the polytope. This concrete description (which they proved only for rational polytopes, ) may shed some light on their geometric meaning. McMullen's recent new proof of the necessity part of the $g$-theorem also gives some hope for elementary interpretation of the $g$-numbers for general polytopes and new proof for their nonnegativity. McMullen's proof also contain a relatively easy reduction from simple polytopes to rational simple polytopes and there is hope that this part, at least, can be extended to general polytopes.

Stanley [46] conjectured that the $g$-vector is an M-vector for every polytope [46]. It is not even known that $g_{k}(P)=0$ implies that $g_{k+1}(P)=0$. Stanley also pointed out what is the extreme combinatorial generality for which the $g$-numbers as defined in this section should work: Namely for regular cell decomposition of (homology) spheres whose faces form a lattice.

For A $d$-polytope $P$, and a subset $S=\left\{i_{1}, \ldots, i_{k}\right\} \subset\{0,1, \ldots, d-1\}$ the flag number $f_{S}(P)$ is the number of chains of faces of $P F_{1} \subset F_{2} \subset \cdots \subset F_{k}$ such that $\operatorname{dim} F_{j}=i_{j}$. (The same definition applies to ranked lattices.) For simplicial polytopes the flag numbers are determined by the face numbers, but for general polytopes flag numbers seems to be the "correct" invariants. A remarkable theorem of Bayer and Billera asserts that the affine dimension of the space of flag numbers of $d$-polytopes is $c_{d}-1$, were $c_{d}$ is the $d$-th Fibonacci number. The $g$-numbers are linear combination of flag numbers and this is explained in details in [1].

\section{3. $g_{2}$ FOR GENERAL POLYTOPES AND ELEMENTARY POLYTOPES}

Let $P$ be a $d$-polytope. A framework based on $P$ is a graph embedded in $R^{d}$ which is obtained by triagulating all the 2-faces of $P$ by polygons. Let $f_{1}^{+}$be the number of edges in such a framework. $g_{2}(P)=f_{1}^{+}(P)-d f_{0}(P)+\left(\begin{array}{c}d+1 \\ 2\end{array}\right)$. Whiteley [51] proved 
(by a clever inductive argument starting with the case $d=3$ which was proved by Alexandrov, ) that every such framework is infinitesimally rigid (i.e. 1-rigid). This implies that $g_{2}(P)$ is the dimension of the space of stresses of a framework based on $P$ and therefore $g_{2}(P)$ is nonnegative for every $d$-polytope.

A polytope $P$ is elementary if $g_{2}(P)=0$.

Theorem 4.1 Let $P$ be a d-polytope then $g_{2}(P) \geq g_{2}(F)+g_{1}(F) g_{1}(P / F)+g_{2}(P / F)$

Proof: (Rough sketch) Let $F$ be a $k$-face of $P$. Choose a vertex in each $(k+1)$-face $G$ containing $F$, which is not in $F$. On all these vertices form a graph $H$ whose edges correspond to edges of a framework of $P / F$. Pur $G=G(F)$. Consider now $G * F$, the join of $G$ and $H$, (Namely, the graph $H \cup G$ union all edges between a vertex in $H$ and a vertex in $G$,) as a framework in $R^{d}$. It is possible to move from $G(P)$ to a framework containing $G * H$ by successively applying the following operation. Move from a framework $A$ to a framework $A^{\prime}$ by adding an edge $e_{1}$ and deleting an edge $e_{2}$ when $A \cup A^{\prime}$ contains a minimal stress containing both $e_{1}$ and $e_{2}$.

Theorem 4.2 (ii) If $g_{2}(P)=0$ then $g_{2}\left(P^{*}\right)=0$.

Proof: (sketch) Every $d$-polytope for $d \leq 3$ is elementary so let $d \geq 4$. For $d=4$ it is easy to verify that $g_{2}(P)=g_{2}\left(P^{*}\right)$. For $d>4$ the Theorem follows by induction using the following:

Theorem 4.3 For a d-polytope $P, d>4$, the following conditions are equivalent

(a) for every proper face $F$ of $P,(1) g_{2}(F)=0$, (2) $g_{2}(P / F)=0$ and (3) Either $F$ is a simplex or $P / F$ is a simplex.

(b) $g_{2}(P)=0$.

Proof: (sketch) (b) implies (a) by Theorem 4.1 above. (a) implies (b) for rational polytopes by the following identity (which can easily be verified by expanding both sides in terms of flag numbers):

$$
\begin{gathered}
3 \sum\left\{g_{2}(F): F \in P_{4}\right\}+2 \sum\left\{g_{1}(F) \cdot g_{1}(P / F): F \in P_{2}\right\} \\
+\sum\left\{g_{2}(P / v): v \in P_{0}\right\}=(d-1) g_{2}(P)+3 g_{3}(P) .
\end{gathered}
$$

(Very rough sketch of the proof for general polytopes is: Follow the second proof of Theorem 9.3 in [27].)

Definition: An abstract polytope is a ranked lattice $L$ such that

(a) every interval of length two has four elements, and

(b) the following connectivity property holds: For every two elements $x$ and $y$ of rank $k$ in $L$ there is a sequence of elements of $\operatorname{rank} k, x=x_{1}, x_{2}, \ldots, x_{t}=y$ such that $\operatorname{rank}\left(x_{i} \wedge x_{i+1}\right)=k-1$ for every $i$ and $x_{i} \leq x \vee y$ for every $i$.

This notion (which is purely combinatorial) clearly includes as special cases simplicial and polyhedral manifolds and pseudomanifolds.

Conjecture 11 The inequality $g_{2} \geq 0$ holds for arbitrary abstract polytopes $L$. Equality holds only for face lattices of polytopes. Moreover, every elementary polytope can be realized with rational coordinates. 
It is conjectured that elementary polytopes have many of the pleasant properties of 3-polytopes. Here is one question in this direction.

Conjecture 12 The graph of every elementary d-polytope is $d+1$ colorable. Moreover, every graph obtained by triangulating (with no new vertices) the 2-faces of an elementary d-polytope is $(d+1)$-colorable.

We also conjecture that the main theorems of this section extend to higher $g_{k}$ 's.

Conjecture $13([29])(1) g_{k}(P)=0$ if and only if $g_{k}\left(P^{*}\right)=0$.

(2) For every face $F, g_{k}(P) \geq \sum_{i=0}^{k} g_{i}(F) g_{k-i}(P / F)$.

\subsection{Diversion: Quotients, Faces And Meisinger's FLAGTOOL}

The reader could have noticed that the inequalities $g_{k} \geq 0$ for general polytopes are rather complicated, and it may be asked to what extent these relations (even if they will be proved completely), are relevant to basic combinatorial properties of polytopes. As described in Bayer's paper [1] (See also [29]) a few basic linear inequalities for flag numbers of polytope imply by convolutions a large number of other inequalities. Günter Meisinger developed a computerized system called FLAGTOOL whose aim is to try to prove automatically theorems on polytopes using the large amount of (known and conjectured) inequalities for face numbers. The following three conjectures were (among others) some targets for FLAGTOOL.

Conjecture 14 (Perles) For every integer $k>0$ there exists $f(k)$ so that every $d$-polytope $d \geq f(k)$ has a $k$-dimensional quotient which is a simplex.

Conjecture 15 For every integer $k>0$ there exist integers $n(k)$ and $d(k)$ so that every $d$-polytope $d \geq d(k)$ has a $k$-dimensional face with at most $n(k)$ vertices.

It can be conjectured that $n(k)$ can be chosen to be $2^{k}$ and that the following stronger conjecture holds

Conjecture 16 ([32]) For every integer $k>0$ there exists $d(k)$ so that every $d$ polytope has a $k$-dimensional face which is either a simplex or combinatorially isomorphic to a cube.

These conjectures are valid for $k=2$, It follows easily from Euler's theorem that every polytope in 3-space has a triangular face or its dual has such a face. It also follows from Euler's theorem that every polytope in 3-space has a face with at most five vertices, and in [32] it is proved that every $d$-polytope, $d \geq 5$ has a face with at most four vertices.

The hope (which was fulfilled) was that FLAGTOOL will automatically prove some of these conjectures in low dimensions and moreover (this was not fulfilled yet) will give some insight on what is involved in a proof for arbitrary dimension.

FLAGTOOL proved automatically the following partial results and supported results to the above conjectures (among many other results). 
Theorem 4.4 (Meisinger [39]) 1. Every rational d-polytope, $d \geq 9$ has a 3-face with at most 150 vertices.

2. Every d-polytope $d \geq 9$, has a 3-dimensional quotient which is a simplex.

3. Every d-polytope $d \geq 7$ has a triangle as the quotient of 1-face in an 4-face.

4. Every 7-polytope has a 3-face with at most 17 vertices or its dual has such a face.

5. Every 5-polytope has a 3-quotient with at most 8 vertices and every 7-polytope has a 4-quotient with at most 16 vertices.

\subsection{Fake $f$-Vectors And more Linear ineQualities}

Every linear combination of face numbers which is nonnegative for all simplicial polytopes is a linear combination with nonnegative coefficients of $g_{0}, g_{2}, \ldots, g_{[d / 2]}$. In [29] the author conjectured that the nonnegativity of $g_{1}, \ldots, g_{[d / 2]}$ give by convolutions (see [1]) all linear inequalities among flag numbers of polytopes. Meisinger [39] showed that this is false and, in fact, if we write $g_{1}[r](P)=f_{r}(P)-\left(\begin{array}{c}d+1 \\ r\end{array}\right)$, then while $g_{1}[r]$ is nonnegative for every $d$-polytope this inequality does not follows from the nonnegativity of the $g_{i}^{\prime} s$. So the problem of finding all linear inequalities for flag numbers of polytopes is wide open. We will describe in this section some conjectures about new such inequalities.

We suspect that the lower bound inequalities which for simplicial polytopes are consequences of the nonnegativity of $g_{2}$ correspond to independent inequalities for general polytopes. We state now what seems to be the right "analogs" of the lower bound inequalities for general polytopes and present a general conjecture which corresponds to the generalized lower bound inequalities.

Let $g_{k}^{r}(P)=\sum\left\{g_{k}(F): F \in P_{k}\right\}$. Thus $g_{0}^{r}(P)=f_{r}(P)$. Recall that $\phi_{k}(n, d)$ is the number of $k$-faces of stacked $d$-polytopes with $n$ vertices. Here is an extension of the lower bound inequalities for general polytopes. There is some hope that certain rigidity type argument may be useful for a proof.

Conjecture 17 Let $P$ be a d-polytope (and more generally an abstract polytope). Then for $k<d-1$,

$$
f_{k}(P)+g_{1}^{k}(P)+g_{1}^{k+1}(P) \geq \phi_{k}(n, d)
$$

and for $k=d-1$,

$$
f_{d-1}(P)+g_{1}^{d-1}(P) \geq \phi_{d-1}(n, d) .
$$

Equality holds if and only if $P$ is an elementary polytope.

Note that the case $k=1$ is just the nonnegativity of $g_{2}(P)$.

We will describe now a more general conjecture. Let $P$ be a simplicial polytope with $g_{k}(P)=0$. For the class of all such polytopes all face numbers are determined by $f_{0}(P), \ldots, f_{k-1}(P)$, and define $\alpha_{k}(r, i)$ such that for simplicial $d$-polytopes with vanishing $g_{k}$ we have $f_{r}=\sum_{i=0}^{k} \alpha_{k}(r, i) f_{i-1} \cdot\left(\alpha_{k}(r, i)\right.$ is determined uniquely.) For arbitrary simplicial $d$-polytopes $P$ one gets the inequalities

$$
f_{r} \geq \sum_{i=0}^{k} \alpha_{k}(r, i) f_{i-1} .
$$


In the simplicial case, thses inequalities do not contribute anything new. They follow from the nonnegativity of the $g$-numbers. Moreover, similar to the case for the lower bound inequalities they also follow from the nonnegativity of the $g_{i}$ 's $i<r$ for the polytope and its quotients.

Remark: In order to get the $\alpha_{k}(r, i)$ explicitly first expend $f_{r}$ in term of the $h_{i}$ 's, by the defining relations. Next use the relations $h_{i} \geq h_{k}$ for $i, d-k \geq i \geq k$, and $h_{i}=h_{d-i}$ for $i>d-k$. This gives an inequality $f_{r} \geq \sum_{i=0}^{k} \beta_{k}(r, i) h_{i}$. Finally expand the $h_{i}$ 's back in term of the $f_{i}^{\prime} s$ to obtain $f_{r} \geq \sum_{i=0}^{k} \alpha_{k}(r, i) f_{i-1}$.

We will consider now general polytopes:

Definition: Let $P$ be an arbitrary $d$-polytope and let $h(P)=\left(h_{0}, h_{1}, \ldots, h_{d}\right)$ be the $h$-vector of $P$. Define the fake $f$-vector of $P$ by $\sum \hat{f}_{k-1}(P)(x-1)^{d-k}=\sum h_{k} x^{d-k}$.

Explicitly one gets

$$
\hat{f}_{k}=\sum_{i=0}^{k} \sum_{r=0}^{i}\left(\begin{array}{l}
i \\
r
\end{array}\right) g_{i}^{k+r} .
$$

Note that the "fake number of edges", $\hat{f}_{1}$ is the number of edges in a framework based on $P$ (denoted before by $f_{1}^{+}(P)$ ).

Now define a truncated version of the fake face number:

$$
\hat{f}_{k}[m]=\sum_{i=0}^{m} \sum_{r=0}^{i}\left(\begin{array}{l}
i \\
r
\end{array}\right) g_{i}^{k+r} .
$$

\section{Conjecture 18}

$$
\hat{f}_{r}[k] \geq \sum_{i=0}^{k} \alpha_{k}(r, i) \hat{f}_{i}
$$

Remark: Another class of conjectures for linear inequalities of flag numbers of polytopes was suggested by Stanley. Let $P$ be an Eulerian poset of rank $n$. The cd-index [3] of $P$ associates for every word $w$ in noncommuting variables $c$ and $d$ such that the number of $c$ 's plus twice the number of $d$ 's is $n$ a certain linear combination of flag numbers of $P$ denoted by $\Phi_{P}(w)$. Stanley conjectured that over all $P$ 's which are face-lattices of $(n-1)$-dimensional polytopes, the value of this linear combination of flag numbers is minimized precisely when $P$ is a Boolean algebra (i.e., the face lattice of a simplex).

\subsection{Centrally symmetric polytopes, Cubical polytopes, Kupitopes and OTHER CLASSES OF POLYTOPES}

It is of interest to study the combinatorial structure of polytopes in special classes of polytopes. We decribe here a few such classes.

- The class of polytopes which were studied the most are the class of centrally symmetric polytopes. There are known lower bound theorems for simplicial centrally symmetric polytopes [45]. But the proofs are non elementary and do not extend to more general structures. For general centrally symmetric polytopes there are some partial results [21]. But even the simple question: Are there always at least $3^{d}$ proper faces is open [31]. For more information see [1]. 
- Kupitopes are polytopes with no triangular 2-faces. Kupitz studied this class of polytopes and conjectured that the number of $r$-faces is at least the number of $r$-faces of the cube. It took quite a while before Blind and Blind [18] proved Kupitz' conjecture. It seem plausible that in analogy with inequality $g_{2}(P) \geq 0$ for general polytopes,

Conjecture 19 For every d-kupitope $P$

$$
f_{1}+1 / 2 \sum\left\{f_{0}(F)-4: F \in P_{2}\right\} \geq(d+1) / 2 f_{0}-2^{d-1} .
$$

In particular, for every cubical d-polytope

$$
f_{1} \geq(d+1) / 2 f_{0}-2^{d-1} .
$$

Some variants of rigidity theory may be helpful here. This is part of a general concept of $h$-numbers for cubical polytopes and kupitopes introduced by Adin $[5]$.

- Polytopes without $r$-faces which are simplices. This may be useful for the study of Conjectures 15, 16.

- A class $\mathcal{M}$ of polytopes which are of interest is the class of polytopes defined by system of linear inequalities each of which have the form $x_{i} \leq a x_{j}+b$. In the context of linear programming these classes were studied by Megiddo [40] and others. But it seems that their combinatorial structure was not studied. Faces of polytopes in $\mathcal{M}$ are also in $\mathcal{M}$.

- Balanced $d$-polytopes of type $\left(k_{1}, k_{2}, \ldots, k_{t}\right)$ are simplicial polytopes whose ertices can be colored with $t$ colors such that each facet contains exactly $k_{i}$ vertices of colors $i$. Of particular interest are balanced polytope of type $(1,1, \ldots, 1)$ which are called completely balanced. Duals of completely balanced polytopes are precisely the simple polytopes with 2-chromatic graphs, or in other words, precisely the simple polytopes all whose 2 -faces have even sides. For an extension of $h$-vector theory to this setting see [44].

- Charney and Davis considered simplicial complexes with no empty simplices of dimension greater than 1 , and called them flag complexes. they made exciting conjectures concerning face numbers of flag polytopes and spheres. (see [19]).

- Another class of polytopes which are of interest are polytopes with the property that every $k$-face has at most $C k$ facets.

\section{7. $h$-VECTORS FOR MORE EXOTIC STRUCTURES}

As we saw $h$-vectors and $g$-vectors plays a crucial role in the study of polytopes and related combinatorial structures. It was suggested that these concept can be extended to much more general classes of combinatorial objects. The extension from simplicial polytopes to general polytopes is instructive. What needed is to add extra terms measuring the amount for which the faces are not simplices.

One direction would be to define $h$-vectors for arbitrary simplicial manifolds and even pseudomanifolds. For manifolds one can expect that the "correcting terms" will be in terms of the Betti numbers. (See [27].) For pseudomanifolds we can expect some terms for Betti numbers of links of faces.

Another direction proposed by Bjorner [14] is to give a definition for arbitrary regular cell decomposition of spheres. The definition and properties of $h$-vectors 
of general polytopes is expected to apply for regular cell decomposition of spheres whose faces form a lattice. For regular cell decomposition of spheres without the lattice property one expects some correction terms for the non lattice property, but so far nobody was able to come up with a reasonable definition even for $h_{1}$. Such $h$-vectors should include as special cases the Kazhdan-Lusztig polynomials.

$h$-vectors play in the combinatorial theory of structures considered here similar role to the role of zeta functions in number theory. (This is not a totally artificial analogy since in some special cases the generating function of the $h$ numbers is a zeta function of some variety.) In simple cases the definition is obvious but proving the basic properties is hard. In more general cases the main challenge is to find the right definition.

\section{References}

1. M. Bayer, Face numbers and subdivisions of convex polytopes, THIS VOLUME pp.

2. C. Lee, Generalized stresses and motions, THIS VOLUME pp.

3. R. Stanley, A Survey of Eulerian Posets, THIS VOLUME, pp.

4. R. Adin, Combinatorial structure of simplicial complexes with symmetry, Ph.D Thesis, Jerusalem, 1991

5. R. Adin, Cubical h-vectors, preprint.

6. A. Altshuler and M. Perles, Quotient polytopes of cyclic polytopes, Part I: Structure and characterization. Isr. J. Math., 36(1980), 97-125.

7. D. Barnette, A proof of the lower bound conjecture for convex polytopes, Pac. J. Math. 46 (1971), 349-354.

8. M. M. Bayer and L. J. Billera, Generalized Dehn-Sommerville relation for polytopes, spheres and Eulerian partially ordered sets, Invent. Math. 79 (1985), 143-157.

9. M. Bayer and A. Klapper, A new index for polytopes, Discrete Comp. Geometry 6(1991), $33-47$.

10. A. Bezdek, K. Bezdek, E. Makai and P. McMullen, Facets with fewest vertices, Monastach Math. 109(1990), 89-96.

11. I.Barany and L. Lovasz, Borsuk's theorem and the number of facets of centrally symmetric polytopes, Acta Math. Acad. Sci. Hung. 40(1982), 323-329.

12. L. J. Billera and C. W. Lee, A proof of the sufficiency of McMullen's conditions for $f$-vectors of simplicial convex polytopes, J. Combin. Th. Ser. A 31 (1981), 237-255.

13. A. Björner, On complements in lattices of finite length, Discrete Math. 36(1981), 325-326.

14. A. Björner, Face numbers of complexes and polytopes, Proc. International Congress of Math., Berkeley, 1986.

15. A. Björner and G. Kalai, Extended Euler Poincare relations, Acta Math. 161 (1988), 279-303.

16. A. Björner and G. Kalai, On $f$-vectors and homology, Combinatorial Mathematics (G.S. Bloom, R.L. Graham and J. Malkevitch, eds.) Proc. NY Academy of Science 555(1989), 63-80.

17. A. Björner and G. Kalai, Extended Euler Poincare relations for regular cell complexes, in: Applied Geometry and Discrete Mathematics, The Klee Festschrift, DIMACS Series in Discrete Mathematics and Computer Science 4(1991),

18. G. Blind and R. Blind, Convex polytopes without triangular faces, Isr. J. Math. 71(1990), 129-134.

19. R. Charney and M. Davis, The Euler characteristics of nonpositively curved, piecewise linear Euclidean manifolds, preprint.

20. P. Erdös and R. Rado, Intersection theorems for system of sets, J. London Math. Soc. 35(1960), 85-90.

21. T. Figiel, J. Lindenstrauss and V. Milman, The dimension of almost spherical sections of convex bodies, Acta Math. 139(1977), 53-94.

22. J.Goodman and R. Pollack, There are asymptotically far fewer polytopes than we thought, Bull. Amer. Math. Soc. 14(1986), 127-129.

23. M. Gromov, Structures métriques pour les variétés Riemanniens, Paris, CEDEX, 1981. 
24. B. Grünbaum, Convex Polytopes, Wiley Interscience, London, 1967.

25. G. Kalai, A characterization of $f$-vectors of families of convex sets in $R^{d}$, Part I: Necessity of Eckhoff's conditions, Israel J. Math. 48 (1984), 175-195.

26. G. Kalai, Open Problems: Collected open problems Oberwolfach Convexity meeting, 1986.

27. G. Kalai, Rigidity and the lower bound theorem I, Invent. Math. 88(1987), 125-151.

28. G. Kalai, Many triangulated spheres, Discrete and Computational Geometry, 3(1988), 1-14.

29. G. Kalai, A new basis of polytopes, J. Comb. Th. (Ser. A), 49(1988), 191-209.

30. G. Kalai, A simple way to tell a simple polytope from its graph, J. Comb. Th. (Ser. A) 49(1988), 381-383.

31. G. Kalai, On the number of faces of centrally-symmetric convex polytopes (research problem), Graphs and Combinatorics, 5 (1989), 389-391.

32. G. Kalai, On low-dimensional faces that high-dimensional polytopes must have, Combinatorica 10(1990), 271-280.

33. G. Kalai, The diameter of graphs of convex polytopes and f-vector theory, in: Applied Geometry and Discrete Mathematics, The Klee Festschrift, DIMACS Series in Discrete Mathematics and Computer Science 4(1991), 387-411.

34. G. Kalai, Algebraic shifting, to appear in Proc. Jerusalem Combinatorics Conference.

35. V. Klee, A combinatorial analogue of Poincaré's duality theorem, Can. J. Math. 16(1964), 517-531.

36. P. McMullen, The number of faces of simplicial polytopes, Israel J. Math. 9(1971), 559-570.

37. P. McMullen, On Simple Polytopes, Inven. Math. 113(1993), 419-444.

38. P. McMullen and D. Walkup, A generalized lower bound conjecture for convex polytopes, Mathematica 18 (1971), 264-273.

39. G. Meisinger, Flag Numbers and Quotients of Convex Polytopes, Ph.D Thesis, Passau University, 1993.

40. N. Megiddo, Toward a genuinely polynomial algorithm for linear programming, SIAM J. Comp. 12(1983), 347-353.

41. M. Perles, Truncation of atomic lattices, unpublished manuscript, around 1970

42. R. Stanley, The upper bound conjecture and Cohen-Macaulay ring, Stud. Appl. Math. 54(1975), 135-142.

43. R. Stanley, The number of faces of simplicial convex polytopes, Adv. Math. 35(1980), 236238 .

44. R. Stanley, Balanced Cohen-Macaulay complexes, Trans. Amer. Math. Soc., 249, (1979), $139-157$.

45. R. Stanley, On the number of faces of centrally symmetric simplicial polytopes, Graphs and Combinatorics 3(1987), 55-66.

46. R. Stanley, Generalized $h$-vectors, intersection cohomology of toric varieties, and related results, in Commutative Algebra and Combinatorics, (M. Nagata and H. Matsumura, eds.), Advanced Studies in Pure Mathematics 11, Kinokuniya, Tokyo, and North-Holland, Amsterdam/New York, 1987, pp. 187-213.

47. R. Stanley, A monotonicity property of h-vectors and h*-vectors, European J. Combin. 14 (1993), 251-258.

48. R. Stanley, Subdivisions and local h-vectors, J. Amer. Math. Soc., 5(1992), 805-851.

49. R. Stanley, Flag vectors and the CD-index, Math. Z., to appear.

50. T-S Tay, N. White and W. Whiteley, Skeletal rigidity of cell complexes, to appear.

51. W. Whiteley, Infinitesimal rigid polyhedra I. Statics of frameworks, Trans. Amer. Math. Soc. 285(1984), 431-465.

52. Günter Ziegler, Lectures on Polytopes, Springer, to appear 